\title{
New Entropy, Similarity Measure of Intuitionistic Fuzzy Sets and their Applications in Group Decision Making*
}

\author{
Xia Liang ${ }^{1}$, Cuiping $\mathrm{Wei}^{2 \dagger}$, Meimei Xia ${ }^{3}$ \\ ${ }^{1}$ School of Business Administration, Northeastern University, \\ Shenyang, Liaoning 110819, China \\ E-mail: susanliangxia@163.com \\ ${ }^{2}$ Institute of Operations Research, Qufu Normal University, \\ Rizhao, Shandong 276826, China \\ E-mail: wei_cuiping@yahoo.com.cn \\ ${ }^{3}$ School of Economics and Management, Tsinghua University, \\ Beijing 100084, China \\ E-mail:meimxia@163.com
}

Received 15th March 2012

Accepted 16th August 2012

\begin{abstract}
In this paper, we propose a new entropy measure with geometrical interpretation of intuitionistic fuzzy sets. Compared with the entropy measure provided by Szmidt and Kacprzyk, the new entropy formula in this paper can measure both fuzziness and intuitionism for intuitionistic fuzzy sets. According to the relationship between entropy and similarity measure, we construct a new similarity measure for intuitionistic fuzzy sets. Then we present two methods, based on entropy and similarity measure, to determine weights of experts for multi-attribute group decision making with intuitionistic fuzzy information.
\end{abstract}

Keywords: multi-attribute group decision making, intuitionistic fuzzy sets, entropy, similarity measure

\section{Introduction}

As a generalized form of fuzzy sets (FSs) ${ }^{1}$, intuitionistic fuzzy sets (IFSs) ${ }^{2}$, characterized by membership functions and non-membership functions, can depict the fuzziness and uncertainty of objective world more exquisitely than fuzzy sets ${ }^{3,4}$.
Zadeh $^{5}$, Gau and Buehrer ${ }^{6}$ introduced the notion of interval-valued fuzzy sets (IVFSs) and vague sets. It was proved that IVFSs and vague sets are equivalent to IFSs ${ }^{7-9}$. Now, IFSs have been applied in various fields, such as decision making ${ }^{10-13}$, medical diagnosis ${ }^{14}$, pattern recognition ${ }^{15,16}$ and clustering ${ }^{17}$.

*The work was partly supported by the National Natural Science Foundation of China $(71171187,71271050)$, Ministry of Education Foundation of Humanities and Social Sciences (10YJC630269).

†Corresponding author, E-mail: wei_cuiping@yahoo.com.cn(C.P.Wei) 
The notion of entropy was introduced by Zadeh $^{18}$, which is used for estimating the fuzziness of fuzzy sets. After that, De Luca and Termini ${ }^{19}$ proposed the axioms with which the fuzzy entropy should comply, and defined a non-probabilistic entropy for IFSs based on Shannon's function. Burillo and Bustince ${ }^{20}$ introduced the definition of entropy for IFSs and IVFSs which can measure the intuitionism degree of an IFS or IVFS. Szmidt and Kacprzyk $^{21}$ extended the definition of fuzzy entropy provided by De Luca and Termini and gave an axiomatic definition of entropy for IFSs. Based on the geometrical interpretation of IFSs, they also proposed a new entropy measure. Vlachos and Sergiadis ${ }^{15}$ derived an entropy formula from a cross entropy of IFSs. They pointed out that entropy of IFSs could measure both fuzziness and intuitionism of an IFS. Many scholars also proposed different entropy formulas for IFSs, IVFSs and vague sets ${ }^{22-26}$.

Similarity measure, as another important topic in the theory of fuzzy sets, has been studied by many scholars. The similarity measure indicates the similar degree between two IFSs. $\mathrm{Li}$ and $\mathrm{Cheng}^{27}$ presented the axiomatic definition of the similarity measure for IFSs. Liang and $\mathrm{Shi}^{28}$ proposed several similarity measures for IFSs and discussed the relationship between these measures. $\mathrm{Xia}$ and $\mathrm{Xu}^{29}, \mathrm{Xu}$ and Yager $^{30}$ defined some similarity measures of intuitionistic fuzzy sets and used them to group decision making. Li et al. ${ }^{31}$ made a comparative analysis of existing similarity measures for IFSs and illustrated some counter-intuitive cases of each measure. $\mathrm{Xu}^{32}$ made a comprehensive overview of similarity measures for IFSs and proposed several similarity measures by different distance measures.

Many researchers have investigated the relationship between entropy and similarity measure. Zeng and $\mathrm{Li}^{33}$, Zhang et al. ${ }^{34}$ proved some theorems that entropy and similarity measure can be transformed by each other. Zeng and $\mathrm{Guo}^{35}$ discussed the relationship of normalized distance, similarity measure, inclusion measure and entropy measure of IVFSs. Wei and Wang ${ }^{36}$ gave an approach to construct similarity measures using entropy for interval-valued intuitionistic fuzzy sets (IVIFSs) and proposed new similarity measures for IFSs and IVIFSs.
Szmidt and Kacprzyk ${ }^{26}$ proposed an entropy measure with geometrical interpretation of IFSs to measure the fuzziness of an IFS. In fact, the uncertainty degree of an IFS includes fuzziness and intuitionism. The fuzziness is dominated by deviation of the membership degree and non-membership degree, while the intuitionism is associated with hesitancy degree ${ }^{22}$. However, the entropy measure provided by Szmidt and Kacprzyk ${ }^{26}$ can not distinguish the uncertainty degree between two different IFSs when they have the same deviations of membership degrees and non-membership degrees. In this paper, we propose a new entropy measure by the geometrical interpretation of IFSs. The new formula can measure not only the fuzziness but also the intuitionism of an IFS.

We organize this paper as follows. Firstly, Section 2 reviews some concepts that will be used in this work. In Section 3, we make a discussion on two existing entropy measures which are introduced by Szmidt ${ }^{26}$ and Vlachos ${ }^{15}$. The entropy provided by Szmidt and Kacprzyk ${ }^{26}$ only describes the fuzziness of IFSs. Then we give a new entropy measure which can adequately describe the fuzziness and intuitionism of an IFS. By investigating the transformation of an entropy into similarity measure, we establish a similarity measure for IFSs in Section 4. Then numerical examples are given to show the rationality of this new similarity measure. In Section 5 , the new entropy and similarity measure are applied to determining weights of experts for intuitionistic fuzzy group decision making. Conclusions are given in Section 6.

\section{Preliminaries}

Definition 1. ${ }^{2}$ Let $X$ be a universe of discourse. An intuitionistic fuzzy set in $X$ is an object having the form:

$$
A=\left\{\left\langle x, \mu_{A}(x), v_{A}(x)\right\rangle \mid x \in X\right\}
$$

where

$$
\mu_{A}: X \rightarrow[0,1], v_{A}: X \rightarrow[0,1]
$$

with the condition

$$
0 \leqslant \mu_{A}(x)+v_{A}(x) \leqslant 1, \forall x \in X .
$$


The numbers $\mu_{A}(x)$ and $v_{A}(x)$ denote the degree of membership and non-membership of $x$ to $A$, respectively.

For each IFS $A$ in $X$, we call $\pi_{A}(x)=1-\mu_{A}(x)-$ $v_{A}(x)$ the intuitionistic index of $x$ in $A$, which denotes the hesitancy degree of $x$ to $A$.

Definition 2. ${ }^{2}$ Let $A=\left\{\left\langle x, \mu_{A}(x), v_{A}(x)\right\rangle \mid x \in X\right\}$ and $B=\left\{\left\langle x, \mu_{B}(x), v_{B}(x)\right\rangle \mid x \in X\right\}$ be two IFSs, their relations and operations are defined as follows:

(1) $A^{C}=\left\{\left\langle x, v_{A}(x), \mu_{A}(x)\right\rangle \mid x \in X\right\}$,

(2) $A \subseteq B$ if and only if $\mu_{A}(x) \leqslant \mu_{B}(x), v_{A}(x) \geqslant$ $v_{B}(x)$, for each $x \in X$,

(3) $A=B$ if and only if $A \subseteq B$ and $B \subseteq A$.

In the rest, we assume that the universe $X$ is a finite set, denoted by $X=\left\{x_{1}, x_{2}, \ldots, x_{n}\right\}$. Let $I F S(X)$ be the set of all the IFSs in $X$.

Definition 3. ${ }^{37}$ Let $A=\left\{\left\langle x_{i}, \mu_{A}\left(x_{i}\right), v_{A}\left(x_{i}\right)\right\rangle \mid x_{i} \in\right.$ $X\}$ and $B=\left\{\left\langle x, \mu_{B}\left(x_{i}\right), v_{B}\left(x_{i}\right)\right\rangle\right.$

$\left.\mid x_{i} \in X\right\}$ be two IFSs. The normalized Hamming distance between $A$ and $B$ is given as follows:

$$
\begin{aligned}
d(A, B) & =\frac{1}{2 n} \sum_{i=1}^{n}\left(\left|\mu_{A}\left(x_{i}\right)-\mu_{B}\left(x_{i}\right)\right|\right. \\
& \left.+\left|v_{A}\left(x_{i}\right)-v_{B}\left(x_{i}\right)\right|+\left|\pi_{A}\left(x_{i}\right)-\pi_{B}\left(x_{i}\right)\right|\right) .(2)
\end{aligned}
$$

For convenience, we call $\alpha=\left(\mu_{\alpha}, v_{\alpha}\right)$ an intuitionistic fuzzy number (IFN) ${ }^{38}$, where $\mu_{\alpha} \in[0,1], v_{\alpha} \in$ $[0,1]$, and $\mu_{\alpha}+v_{\alpha} \leqslant 1$. Let $\Theta$ be the universal set of IFNs.

For comparison of IFNs, Chen and $\operatorname{Tan}^{39}$ defined a score function while Hong and $\mathrm{Choi}^{40} \mathrm{de}$ fined an accuracy function. Based on the two functions, $\mathrm{Xu}^{38}$ provided a method to compare two intuitionistic fuzzy numbers (IFNs).

Definition 4. ${ }^{38}$ Let $\alpha=\left(\mu_{\alpha}, v_{\alpha}\right)$ and $\beta=\left(\mu_{\beta}, v_{\beta}\right)$ be two IFNs, $s(\alpha)=\mu_{\alpha}-v_{\alpha}$ and $s(\beta)=\mu_{\beta}-v_{\beta}$ be the score degrees of $\alpha$ and $\beta$, respectively, $h(\alpha)=$ $\mu_{\alpha}+v_{\alpha}$ and $h(\beta)=\mu_{\beta}+v_{\beta}$ be the accuracy degrees of $\alpha$ and $\beta$, respectively. Then

(1) If $s(\alpha)<s(\beta)$, then $\alpha$ is smaller than $\beta$, denoted by $\alpha<\beta$,

(2) If $s(\alpha)=s(\beta)$, then

1)If $h(\alpha)=h(\beta)$, then $\alpha$ and $\beta$ represent the same information, i.e., $\mu_{\alpha}=\mu_{\beta}$ and $v_{\alpha}=v_{\beta}$, denoted by $\alpha=\beta$,
2) If $h(\alpha)<h(\beta)$, then $\alpha$ is smaller than $\beta$, denoted by $\alpha<\beta$,

3) If $h(\alpha)>h(\beta)$, then $\alpha$ is bigger than $\beta$, denoted by $\alpha>\beta$.

Definition 5. ${ }^{41}$ Let $\alpha=\left(\mu_{\alpha}, v_{\alpha}\right)$ and $\beta=$ $\left(\mu_{\beta}, v_{\beta}\right)$ be two IFNs. Then three operational laws of IFNs are given as follows:

(1) $\alpha \oplus \beta=\left(\mu_{\alpha}+\mu_{\beta}-\mu_{\alpha} \mu_{\beta}, v_{\alpha} v_{\beta}\right)$,

(2) $\lambda \alpha=\left(1-\left(1-\mu_{\alpha}\right)^{\lambda}, v_{\alpha}^{\lambda}\right), \lambda \geqslant 0$,

(3) $\alpha^{c}=\left(v_{\alpha}, \mu_{\alpha}\right)$.

With the thorough research of intuitionistic fuzzy set theory and the continuous expansion of its application scope, it is more and more important to aggregate intuitionistic fuzzy information effectively. $\mathrm{Xu}^{41}$ proposed intuitionistic fuzzy weighted averaging (IFWA) operator to aggregate the intuitionistic fuzzy information.

Definition 6. ${ }^{41}$ Let $\alpha_{i}=\left(\mu_{\alpha_{i}}, v_{\alpha_{i}}\right)(i=1,2, \cdots, n)$ be a collection of IFNs. An intuitionistic fuzzy weighted averaging (IFWA) operator is a mapping: $\Theta^{n} \rightarrow \Theta$, such that

$$
\begin{aligned}
\operatorname{IFWA}\left(\alpha_{1}, \alpha_{2}, \cdots, \alpha_{n}\right) & =w_{1} \alpha_{1} \oplus w_{2} \alpha_{2} \oplus \cdots \oplus w_{n} \alpha_{n} \\
& =\left(1-\prod_{j=1}^{n}\left(1-\mu_{\alpha_{j}}\right)^{w_{j}}, \prod_{j=1}^{n} v_{\alpha_{j}}^{w_{j}}\right)
\end{aligned}
$$

where $w=\left(w_{1}, w_{2}, \cdots, w_{n}\right)^{T}$ is the weighting vector of $\alpha_{i}(i=1,2, \cdots, n)$ with $w_{j} \in[0,1]$ and $\sum_{j=1}^{n} w_{j}=1$.

\section{Entropy for intuitionistic fuzzy sets}

Szmidt and Kacprzyk ${ }^{21}$ generalized the notion of fuzzy entropy proposed by De Luca and Termini ${ }^{19}$ and introduced the following axiomatic definition of entropy for IFSs.

Definition 7. 21 A real-valued function $E$ : $\operatorname{IFS}(X) \rightarrow[0,1]$ is called an intuitionistic fuzzy entropy on $\operatorname{IFS}(X)$ if it satisfies the following axiomatic requirements:

(E1) $E(A)=0$ if and only if $A$ is a crisp set,

(E2) $E(A)=1$ if and only if $\mu_{A}\left(x_{i}\right)=v_{A}\left(x_{i}\right)$, for each $x_{i} \in X$,

(E3) $E(A) \leqslant E(B)$ if $A \subseteq B$ when $\mu_{B}\left(x_{i}\right) \leqslant v_{B}\left(x_{i}\right)$ or $B \subseteq A$ when $\mu_{B}\left(x_{i}\right) \geqslant v_{B}\left(x_{i}\right)$ for each $x_{i} \in X$, 
(E4) $E(A)=E\left(A^{C}\right)$.

\subsection{Discussion on existing entropy measures for IFSs}

In the following, we investigate geometrical representation of IFSs. For each element $x=\left(\mu_{x}, \nu_{x}, \pi_{x}\right)$ belonging to an IFS which such that $\mu_{x}+v_{x}+\pi_{x}=$ 1 , where $\mu_{x}, v_{x}, \pi_{x} \in[0,1]$. We can imagine a unit cube (Figure 1) inside which there is $A B D$ triangle where the above equations are fulfilled. In other words, each element belonging to an IFS can be represented as a point $(\mu, v, \pi)$ inside the triangle $A B D$.

Motivated by the geometrical representation of IFSs, Szmidt and Kacprzyk ${ }^{26}$ defined entropy $E(F)$ for a separate element $F$ (represented by point in Figure 2).

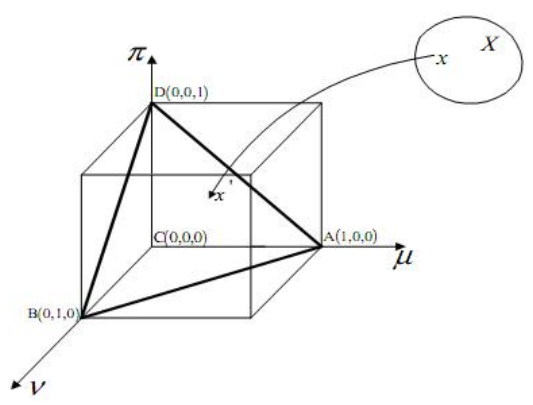

Figure 1: Geometrical representation

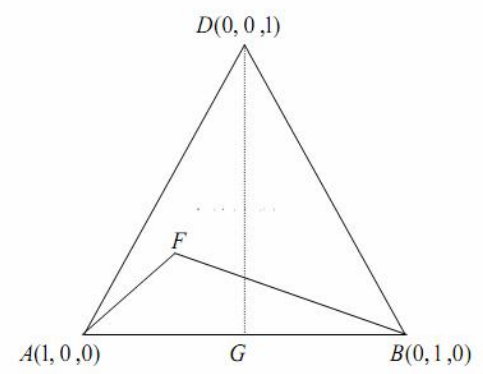

Figure 2: The triangle $A B D$ (cf. Fig. 1)

Definition 8. 26 The entropy of element $F\left(\mu_{F}, \nu_{F}, \pi_{F}\right)$ belonging to an IFS is as follows:

$$
\begin{aligned}
E(F) & =1-\frac{1}{2}\left[d\left(F, F_{\text {far }}\right)-d\left(F, F_{\text {near }}\right)\right] \\
& =1-\frac{1}{2}\left|\mu_{F}-v_{F}\right|
\end{aligned}
$$

where $A\left(\mu_{A}, v_{A}, \pi_{A}\right)=(1,0,0), B\left(\mu_{B}, v_{B}, \pi_{B}\right)=$ $(0,1,0), d\left(F, F_{\text {near }}\right)$ is a distance from $F$ to the nearer point $F_{\text {near }}$ among $A$ and $B, d\left(F, F_{\text {far }}\right)$ is the distance from $F$ to the farer point $F_{f a r}$ among $A$ and $B$, $d\left(F, F_{\text {far }}\right)$ and $d\left(F, F_{\text {near }}\right)$ are obtained by Formula (2).

Formula (4) describes entropy for a single element belonging to an IFS. For $n$ elements belonging to IFS A, Szmidt and Kacprzyk ${ }^{21}$ defined an entropy of $A$ :

$$
E_{S K}(A)=1-\frac{1}{2 n} \sum_{i=1}^{n}\left|\mu_{A}\left(x_{i}\right)-v_{A}\left(x_{i}\right)\right| .
$$

Vlachos and Sergiadis ${ }^{15}$ pointed out that fuzzy entropy describes the fuzziness of FSs. Since the theory of IFSs is a generalization of that of FSs, intuitionistic fuzzy entropy should measure both the fuzziness and intuitionism for IFSs.

Vlachos and Sergiadis ${ }^{15}$ induced an entropy measure $E_{V S}$ based on a cross entropy measure of IFSs:

$$
\begin{aligned}
E_{V S}(A) & =-\frac{1}{n \ln 2} \sum_{i=1}^{n}\left[\mu_{A}\left(x_{i}\right) \ln \mu_{A}\left(x_{i}\right)+v_{A}\left(x_{i}\right) \ln v_{A}\left(x_{i}\right)\right. \\
& \left.-\left(1-\pi_{A}\left(x_{i}\right)\right) \ln \left(1-\pi_{A}\left(x_{i}\right)\right)-\pi_{A}\left(x_{i}\right) \ln 2\right] .(6)
\end{aligned}
$$

The Formula (6) can be rewritten as $E_{V S}(A)=$ $E_{\text {fuzz }}(A)+E_{\text {intuit }}(A)$, where

$$
\begin{aligned}
E_{f u z z}(A) & =-\frac{1}{n \ln 2} \sum_{i=1}^{n}\left[\mu_{A}\left(x_{i}\right) \ln \mu_{A}\left(x_{i}\right)+v_{A}\left(x_{i}\right) \ln v_{A}\left(x_{i}\right)\right. \\
& \left.-\left(1-\pi_{A}\left(x_{i}\right)\right) \ln \left(1-\pi_{A}\left(x_{i}\right)\right)\right], \\
E_{\text {intuit }}(A) & =\frac{1}{n} \sum_{i=1}^{n} \pi_{A}\left(x_{i}\right) . \quad E_{f u z z}(A) \text { expresses the }
\end{aligned}
$$
fuzziness degree of $A$, while $E_{\text {intuit }}(A)$ expresses the intuitionism degree of $A$. Therefore, $E_{V S}$ can measure both the fuzziness and intuitionism for IFSs.

The following example shows that Formula (5) and (6) can produce some counter-intuition cases.

Example 1. Let $A_{1}=\{\langle x, 0.4,0.6\rangle \mid x \in X\}, A_{2}=$ $\{\langle x, 0.3,0.5\rangle \mid x \in X\}$ and $A_{3}=\{\langle x, 0.1,0.3\rangle$ $\mid x \in X\}$ be three IFSs. Using the entropy measures $E_{S K}$ and $E_{V S}$ we get

$$
E_{S K}\left(A_{1}\right)=E_{S K}\left(A_{2}\right)=E_{S K}\left(A_{3}\right)=0.9000,
$$


$E_{V S}\left(A_{1}\right)=0.9710, E_{V S}\left(A_{2}\right)=0.9635, E_{V S}\left(A_{3}\right)=0.9245$.

For IFSs $A_{1}, A_{2}$ and $A_{3}$, we can see that the deviations of their membership degrees and nonmembership degrees are same, but their hesitancy degrees are increasing. Therefore, the uncertainty degrees of $A_{1}, A_{2}$ and $A_{3}$ are increasing. However, by Formula (5), we can derive the same entropies of IFSs $A_{1}, A_{2}$ and $A_{3}$. It is obvious that the results are not so reasonable as we expect. In fact, the formula $E_{S K}$ can measure only the fuzziness degree instead of the intuitionism degree for IFSs.

By formula $E_{V S}$, we know that the entropies of $A_{1}, A_{2}$ and $A_{3}$ are decreasing, which are not consistent with our intuition. Then we can prove the following property of formula $E_{V S}$.

Theorem 1. Let $X=\{x\}$. For a constant a in $(0,1)$, let $\mathscr{F}_{a}$ be the set of all IFSs $\left.\left\{<x, \mu_{A}(x), v_{A}(x)\right\rangle\right\}$ in $X$ with $\left|\mu_{A}(x)-v_{A}(x)\right|=a$. Then $E_{V S}(A)$ is strictly monotone decreasing with respect to $\pi_{A}(x)$ on $\mathscr{F}_{a}$.

Proof. Since $\pi_{A}(x)=1-\mu_{A}(x)-v_{A}(x), \mid \mu_{A}(x)-$ $v_{A}(x) \mid=a(0<a<1), \mu_{A}(x)=\frac{1-\pi_{A}(x)+a}{2}, v_{A}(x)=$ $\frac{1-\pi_{A}(x)-a}{2}$; or $v_{A}(x)=\frac{1-\pi_{A}(x)+a}{2}, \mu_{A}(x)=\frac{1-\pi_{A}(x)-a}{2}$. Thus,

$$
\begin{aligned}
E_{V S}(A) & =-\frac{1}{\ln 2}\left[\frac{1-\pi_{A}(x)+a}{2} \ln \frac{1-\pi_{A}(x)+a}{2}\right. \\
& +\frac{1-\pi_{A}(x)-a}{2} \ln \frac{1-\pi_{A}(x)-a}{2} \\
& \left.-\left(1-\pi_{A}(x)\right) \ln \left(1-\pi_{A}(x)\right)-\pi_{A}(x) \ln 2\right] .
\end{aligned}
$$

Let

$$
\begin{aligned}
f\left(\pi_{A}(x)\right) & =\frac{1-\pi_{A}(x)+a}{2} \ln \frac{1-\pi_{A}(x)+a}{2} \\
& +\frac{1-\pi_{A}(x)-a}{2} \ln \frac{1-\pi_{A}(x)-a}{2} \\
& -\left(1-\pi_{A}(x)\right) \ln \left(1-\pi_{A}(x)\right)-\pi_{A}(x) \ln 2,
\end{aligned}
$$

hence $f^{\prime}\left(\pi_{A}(x)\right)=-\frac{1}{2} \ln \frac{1-\pi_{A}(x)+a}{2}-\frac{1}{2} \frac{1-\pi_{A}(x)-a}{2}+$ $\ln \left(1-\pi_{A}(x)\right)-\ln 2=-\frac{1}{2} \ln \left[\frac{\left(1-\pi_{A}(x)\right)^{2}-a^{2}}{\left(1-\pi_{A}(x)\right)^{2}}\right]$. Since $\pi_{A}(x) \in[0,1]$ and $a \in(0,1), 0 \leqslant \frac{\left(1-\pi_{A}(x)\right)^{2}-a^{2}}{\left(1-\pi_{A}(x)\right)^{2}}<1$. Therefore, $f^{\prime}\left(\pi_{A}(x)\right)>0, f\left(\pi_{A}(x)\right)$ is strictly monotone increasing with respect to $\pi_{A}(x)$ on $\mathscr{F}_{a}$, that is, $E_{V S}(A)$ is strictly monotone decreasing with respect to $\pi_{A}(x)$ on $\mathscr{F}_{a}$.
In the following, we will propose a new entropy measure which can measure both the fuzziness and intuitionism of IFSs.

\subsection{A new entropy measures for IFSs}

Theorem 2. Let $F\left(\mu_{F}, v_{F}, \pi_{F}\right)$ be a separate element belonging to an IFS (represented by point in Figure 2). Then we have $d(F, A)+d(F, B)+$ $d(F, D)=2$.

Proof. Since $A\left(\mu_{A}, v_{A}, \pi_{A}\right)=(1,0,0)$, $B\left(\mu_{B}, v_{B}, \pi_{B}\right)=(0,1,0), D\left(\mu_{D}, v_{D}, \pi_{D}\right)=(0,0,1)$, we can get

$$
\begin{aligned}
& d(F, A)+d(F, B)+d(F, D) \\
= & \frac{1}{2}\left(\left|\mu_{F}-1\right|+v_{F}+\pi_{F}+\mu_{F}+\left|v_{F}-1\right|\right. \\
+ & \left.\pi_{F}+\mu_{F}+v_{F}+\left|\pi_{F}-1\right|\right) \\
= & 2 .
\end{aligned}
$$

$D\left(\mu_{D}, v_{D}, \pi_{D}\right)=(0,0,1)$ is the fuzziest element belonging to an IFS, the nearer from $F$ to $D$, the bigger uncertainty degree of $F$. That is, the bigger $2-d(F, D)$, the bigger uncertainty degree of $F$. From Theorem 2, we know $2-d(F, D)=d(F, A)+$ $d(F, B)$. Therefore, the bigger $d(F, A)+d(F, B)$, the bigger uncertainty degree of $F$. Now we give a new entropy measure for single element $F$ belonging to an IFS.

Definition 9. For any point $F\left(\mu_{F}, v_{F}, \pi_{F}\right)$ belonging to an IFS (represented by point in Figure 2), the entropy of $F$ is defined as follows:

$$
\begin{aligned}
E(F) & =1-\frac{|d(F, A)-d(F, B)|}{d(F, A)+d(F, B)} \\
& =1-\frac{\left|\mu_{F}-v_{F}\right|}{1+\pi_{F}} \\
& =\frac{1-\left|\mu_{F}-v_{F}\right|+\pi_{F}}{1+\pi_{F}}
\end{aligned}
$$

Formula (8) describes entropy for a separate element belonging to an IFS. For $n$ elements belonging to an IFS $A$, we have

$$
E(A)=\frac{1}{n} \sum_{i=1}^{n} \frac{1-\left|\mu_{A}\left(x_{i}\right)-v_{A}\left(x_{i}\right)\right|+\pi_{A}\left(x_{i}\right)}{1+\pi_{A}\left(x_{i}\right)} .
$$


Theorem 3. The mapping E, defined by Formula (9), is an entropy measure for IFSs.

Proof. In order for (9) to be qualified as an entropy measure for intuitionistic fuzzy sets, it must satisfy the conditions $E(1)-E(4)$ in Definition 7.

Let $E_{i}(A)=\frac{1-\left|\mu_{A}\left(x_{i}\right)-v_{A}\left(x_{i}\right)\right|+\pi_{A}\left(x_{i}\right)}{1+\pi_{A}\left(x_{i}\right)}$. From $0 \leqslant$ $\mu_{A}\left(x_{i}\right) \leqslant 1,0 \leqslant v_{A}\left(x_{i}\right) \leqslant 1,0 \leqslant \pi_{A}\left(x_{i}\right) \leqslant 1$, we have $0 \leqslant E_{i}(A) \leqslant 1$.

$(E 1)$ Suppose $E(A)=0$. Since $0 \leqslant E_{i}(A) \leqslant 1$ and $E(A)=\frac{1}{n} \sum_{i=1}^{n} E_{i}(A)$, it follows that $E_{i}(A)=0$. So we have $1-\left|\mu_{A}\left(x_{i}\right)-v_{A}\left(x_{i}\right)\right|+\pi_{A}\left(x_{i}\right)=0$. Thus $\mu_{A}\left(x_{i}\right)=1, v_{A}\left(x_{i}\right)=0$, or $\mu_{A}\left(x_{i}\right)=0, v_{A}\left(x_{i}\right)=1$ for each $x_{i} \in X$. Therefore, $A$ be a crisp set. On the other hand, let $A$ be a crisp set, i.e. $\mu_{A}\left(x_{i}\right)=1, v_{A}\left(x_{i}\right)=0$, or $\mu_{A}\left(x_{i}\right)=0, v_{A}\left(x_{i}\right)=1$ for each $x_{i} \in X$. Now matter in which case, we have $E_{i}(A)=0$. Thus $E(A)=0$.

(E2) Let $E(A)=1$, from $E(A)=\frac{1}{n} \sum_{i=1}^{n} E_{i}(A)$ and $0 \leqslant E_{i}(A) \leqslant 1$, we have $E_{i}(A)=1$. Thus $\mu_{A}\left(x_{i}\right)=v_{A}\left(x_{i}\right)$ for each $x_{i} \in X$. Now suppose that $\mu_{A}\left(x_{i}\right)=v_{A}\left(x_{i}\right)$ for each $x_{i} \in X$. Applying this condition to Formula (9), we yield $E(A)=1$.

(E3) Suppose that $B \subseteq A$ when $\mu_{B}\left(x_{i}\right) \geqslant$ $v_{B}\left(x_{i}\right)$ for each $x_{i} \in X$, that is $\mu_{A}\left(x_{i}\right) \geqslant \mu_{B}\left(x_{i}\right)$ and $v_{B}\left(x_{i}\right) \geqslant v_{A}\left(x_{i}\right)$ when $\mu_{B}\left(x_{i}\right) \geqslant v_{B}\left(x_{i}\right)$ for each $x_{i} \in X$. Since $1-\mu_{A}\left(x_{i}\right) \geqslant 0, v_{A}\left(x_{i}\right)-1 \leqslant 0$, we have $v_{B}\left(x_{i}\right)\left(1-\mu_{A}\left(x_{i}\right)\right) \geqslant v_{A}\left(x_{i}\right)\left(1-\mu_{A}\left(x_{i}\right)\right)$ and $\mu_{B}\left(x_{i}\right)\left(v_{A}\left(x_{i}\right)-1\right) \geqslant \mu_{A}\left(x_{i}\right)\left(v_{A}\left(x_{i}\right)-1\right)$. Hence $v_{B}\left(x_{i}\right)\left(1-\mu_{A}\left(x_{i}\right)\right)+\mu_{B}\left(x_{i}\right)\left(v_{A}\left(x_{i}\right)-1\right) \geqslant$ $v_{A}\left(x_{i}\right)\left(1-\mu_{A}\left(x_{i}\right)\right)+\mu_{A}\left(x_{i}\right)\left(v_{A}\left(x_{i}\right)-1\right)$. It follows that $v_{B}\left(x_{i}\right)\left(1-\mu_{A}\left(x_{i}\right)\right)+\mu_{B}\left(x_{i}\right)\left(v_{A}\left(x_{i}\right)-\right.$ $1)+\mu_{A}\left(x_{i}\right)-v_{A}\left(x_{i}\right) \geqslant 0$. Thus $\left(1-\mu_{A}\left(x_{i}\right)\right)(2-$ $\left.\mu_{B}\left(x_{i}\right)-v_{B}\left(x_{i}\right)\right) \leqslant\left(1-\mu_{B}\left(x_{i}\right)\right)\left(2-\mu_{A}\left(x_{i}\right)-\right.$ $\left.v_{A}\left(x_{i}\right)\right)$, which implies $\frac{1-\left|\mu_{A}\left(x_{i}\right)-v_{A}\left(x_{i}\right)\right|+\pi_{A}\left(x_{i}\right)}{1+\pi_{A}\left(x_{i}\right)} \leqslant$ $\frac{1-\left|\mu_{B}\left(x_{i}\right)-v_{B}\left(x_{i}\right)\right|+\pi_{B}\left(x_{i}\right)}{1+\pi_{B}\left(x_{i}\right)}$. Thus $E_{i}(A) \leqslant E_{i}(B)$ for each $x_{i} \in X$. Similarity, when $A \subseteq B$ when $\mu_{B}\left(x_{i}\right) \leqslant v_{B}\left(x_{i}\right)$ for each $x_{i} \in X$, we can also prove that $E_{i}(A) \leqslant E_{i}(B)$ for each $x_{i} \in X$. Therefore, $E(A) \leqslant E(B)$.

(E4) For $A^{c}=\left\{\left\langle x_{i}, v_{A}\left(x_{i}\right), \mu_{A}\left(x_{i}\right)\right\rangle \mid x_{i} \in X\right\}$, we can easily obtain that

$$
E\left(A^{C}\right)=\frac{1}{n} \sum_{i=1}^{n} \frac{1-\left|v_{A}\left(x_{i}\right)-\mu_{A}\left(x_{i}\right)\right|+\pi_{A}\left(x_{i}\right)}{1+\pi_{A}\left(x_{i}\right)}
$$

$$
=\frac{1}{n} \sum_{i=1}^{n} \frac{1-\left|\mu_{A}\left(x_{i}\right)-v_{A}\left(x_{i}\right)\right|+\pi_{A}\left(x_{i}\right)}{1+\pi_{A}\left(x_{i}\right)}=E(A) .
$$

Now we apply Formula (9) to calculate the entropies of IFSs in Example 1, we have

$E\left(A_{1}\right)=0.8000, E\left(A_{2}\right)=0.8333, E\left(A_{3}\right)=0.8750$.

From the results, we can see that if the deviations of membership degrees and non-membership degrees of IFSs are same, the entropies which calculated by $E$ are increasing with respect to hesitancy degrees of IFSs. In fact, formula $E$ satisfies the following theorem.

Theorem 4. Let $X=\{x\}$. For a constant a in $(0,1]$, let $\mathscr{F}_{a}$ be the set of all IFSs $\left\{\left\langle x, \mu_{A}(x), v_{A}(x)\right\rangle\right\}$ in $X$ with $\left|\mu_{A}(x)-v_{A}(x)\right|=a$. Then $E(A)$ is strictly monotone increasing with respect to $\pi_{A}(x)$ on $\mathscr{F}_{a}$.

Proof. Since $\left|\mu_{A}(x)-v_{A}(x)\right|=a(a \in(0,1])$, $E(A)=\frac{1-\left|\mu_{A}(x)-v_{A}(x)\right|+\pi_{A}(x)}{1+\pi_{A}(x)}=\frac{1-a+\pi_{A}(x)}{1+\pi_{A}(x)}$. Let $f\left(\pi_{A}(x)\right)=\frac{1-a+\pi_{A}(x)}{1+\pi_{A}(x)}$, hence $f^{\prime}\left(\pi_{A}(x)\right)=\frac{a}{\left(1+\pi_{A}(x)\right)^{2}}$. Since $\pi_{A}(x) \in[0,1]$ and $a \in(0,1]$. Therefore, $f^{\prime}\left(\pi_{A}(x)\right)>0, f\left(\pi_{A}(x)\right)$ is strictly increasing with respect to $\pi_{A}(x)$ on $\mathscr{F}_{a}$, that is, $E(A)$ is strictly monotone increasing with respect to $\pi_{A}(x)$ on $\mathscr{F}_{a}$.

Example 2. Let $\left.A_{1}=\{<x, 0.1,0.9\rangle \mid x \in X\right\}, A_{2}=$ $\{<x, 0.1,0.7\rangle \mid x \in X\}, A_{3}=\{<x, 0.2,0.7\rangle$ $\mid x \in X\}, A_{4}=\{<x, 0.2,0.5>\mid x \in X\}, A_{5}=\{<$ $x, 0.2,0.4>\mid x \in X\}, A_{6}=\{\langle x, 0.4,0.5\rangle \mid x \in$ $X\}, A_{7}=\{<x, 0.3,0.4>\mid x \in X\} \quad A_{8}=\{<$ $x, 0.1,0.2>\mid x \in X\}$ and $A_{9}=\{\langle x, 0.3,0.3\rangle \mid x \in$ $X\}$ be nine IFSs. Using the entropies $E_{S K}, E_{V S}$ and $E$, the comparison results are listed in Table 1.

Table 1 Comparison of entropies

with different famulas for IFSs $A_{1}-A_{9}$.

\begin{tabular}{lcccc}
\hline & $A_{1}$ & $A_{2}$ & $A_{3}$ & $A_{4}$ \\
\hline$E_{S K}$ & 0.6000 & 0.7000 & 0.7500 & 0.8500 \\
$E_{V S}$ & 0.4690 & 0.6349 & 0.7878 & 0.9042 \\
$E$ & 0.2000 & 0.5000 & 0.5455 & 0.7692 \\
\hline \hline \multicolumn{1}{c}{$A_{5}$} & $A_{6}$ & $A_{7}$ & $A_{8}$ & $A_{9}$ \\
\hline 0.9000 & $\mathbf{0 . 9 5 0 0}$ & $\mathbf{0 . 9 5 0 0}$ & $\mathbf{0 . 9 5 0 0}$ & 1.0000 \\
0.9510 & $\mathbf{0 . 9 9 2 0}$ & $\mathbf{0 . 9 8 9 7}$ & $\mathbf{0 . 9 7 5 5}$ & 1.0000 \\
0.8571 & 0.9091 & 0.9231 & 0.9412 & 1.0000 \\
\hline
\end{tabular}


As can be seen from Table 1, the numerical examples in bold type reflect some counter-intuition cases with formulas $E_{S K}$ and $E_{V S}$. Therefore, the formula $E$ is more reasonable than $E_{S K}$ and $E_{V S}$ for measuring the uncertainty degrees of IFSs.

\section{Similarity measures for intuitionistic fuzzy sets}

In this section, we induce a new similarity measure based on the new entropy measure proposed in Section 3 . Then we compare the new similarity measure with some existing similarity measures.

$\mathrm{Li}$ and Cheng ${ }^{27}$, Mitchell ${ }^{42}$ proposed the axiomatic definitions of the similarity measure between two IFSs.

Definition 10. ${ }^{27,42}$ A real-valued function $S$ : $\operatorname{IFS}(X) \times \operatorname{IFS}(X) \rightarrow[0,1]$ is called a similarity measure on $\operatorname{IFS}(X)$, if it satisfies the following axiomatic requirements:

(S1) $0 \leqslant S(A, B) \leqslant 1$,

(S2) $S(A, B)=1$ if and only if $A=B$,

(S3) $S(A, B)=S(B, A)$,

(S4) If $A \subseteq B \subseteq C$, then $S(A, C) \leqslant S(A, B)$ and $S(A, C) \leqslant S(B, C)$.

\subsection{A new similarity measure for intuitionistic fuzzy sets}

Zeng and $\mathrm{Li}^{33}$ investigated the relationship between entropy and similarity measure of IVFSs and proved some theorems that entropy and similarity measure can be transformed by each other. According to the equivalence of IVFSs and IFSs ${ }^{8,9}$, we propose a transforming method by which one can establish a similarity measure based on an entropy of IFSs.

Suppose $A=\left\{\left\langle x_{i}, \mu_{A}\left(x_{i}\right), v_{A}\left(x_{i}\right)\right\rangle \mid x_{i} \in X\right\}$ and $B=\left\{\left\langle x_{i}, \mu_{B}\left(x_{i}\right), v_{B}\left(x_{i}\right)\right\rangle \mid x_{i} \in X\right\} \quad$ are two IFSs. Then we define $M(A, B)=$ $\left\{\left\langle x_{i}, \mu_{M(A, B)}\left(x_{i}\right), v_{M(A, B)}\left(x_{i}\right)\right\rangle \mid x_{i} \in X\right\}$, where

$\mu_{M(A, B)}\left(x_{i}\right)=\frac{1+\min \left\{\left|\mu_{A}\left(x_{i}\right)-\mu_{B}\left(x_{i}\right)\right|,\left|v_{A}\left(x_{i}\right)-v_{B}\left(x_{i}\right)\right|\right\}}{2}$,

$v_{M(A, B)}\left(x_{i}\right)=\frac{1-\max \left\{\left|\mu_{A}\left(x_{i}\right)-\mu_{B}\left(x_{i}\right)\right|,\left|v_{A}\left(x_{i}\right)-v_{B}\left(x_{i}\right)\right|\right\}}{2}$.
Obviously, $M(A, B)$ is an IFS. By Ref. 33 and the equivalence of IVFSs and IFSs ${ }^{8,9}$, we can easily get the following theorems.

Theorem 5. Let $E$ be an entropy for IFSs. Then for each pair of IFSs $A$ and $B, E(M(A, B))$ is a similarity measure between $A$ and $B$.

Theorem 6. Let $E$ be an entropy measure defined by formula (9), i.e., for

$$
E(A)=\frac{1}{n} \sum_{i=1}^{n} \frac{1-\left|\mu_{A}\left(x_{i}\right)-v_{A}\left(x_{i}\right)\right|+\pi_{A}\left(x_{i}\right)}{1+\pi_{A}\left(x_{i}\right)},
$$

then the function $S$ defined by

$$
S(A, B)=\frac{1}{n} \sum_{i=1}^{n} \frac{2-2 \min \left\{\left|\mu_{A}\left(x_{i}\right)-\mu_{B}\left(x_{i}\right)\right|, \mid v_{A}\left(x_{i}\right)-v_{B}\left(x_{i}\right)\right\}}{2+|| \mu_{A}\left(x_{i}\right)-\mu_{B}\left(x_{i}\right)|-| v_{A}\left(x_{i}\right)-v_{B}\left(x_{i}\right)||}
$$

is a similarity measure between IFSs $A$ and $B$.

Proof. By the definition of $M(A, B)$, we have

$$
\begin{aligned}
& \left|\mu_{M(A, B)}\left(x_{i}\right)-v_{M(A, B)}\left(x_{i}\right)\right| \\
= & \frac{\max \left\{\left|\mu_{A}\left(x_{i}\right)-\mu_{B}\left(x_{i}\right)\right|,\left|v_{A}\left(x_{i}\right)-v_{B}\left(x_{i}\right)\right|\right\}}{2} \\
+ & \frac{\min \left\{\left|\mu_{A}\left(x_{i}\right)-\mu_{B}\left(x_{i}\right)\right|,\left|v_{A}\left(x_{i}\right)-v_{B}\left(x_{i}\right)\right|\right\}}{2} .
\end{aligned}
$$

The hesitancy degree of $x_{i}$ in $M(A, B)$ is

$$
\begin{aligned}
\pi_{M(A, B)}\left(x_{i}\right) & =1-\mu_{M(A, B)}\left(x_{i}\right)-v_{M(A, B)}\left(x_{i}\right) \\
& =\frac{\max \left\{\left|\mu_{A}\left(x_{i}\right)-\mu_{B}\left(x_{i}\right)\right|,\left|v_{A}\left(x_{i}\right)-v_{B}\left(x_{i}\right)\right|\right\}}{2} \\
& -\frac{\min \left\{\left|\mu_{A}\left(x_{i}\right)-\mu_{B}\left(x_{i}\right)\right|,\left|v_{A}\left(x_{i}\right)-v_{B}\left(x_{i}\right)\right|\right\}}{2} .
\end{aligned}
$$

Let $\left|\mu_{A}\left(x_{i}\right)-\mu_{B}\left(x_{i}\right)\right|=\mu_{i},\left|v_{A}\left(x_{i}\right)-v_{B}\left(x_{i}\right)\right|=v_{i}$; $\max \left\{\mu_{i}, v_{i}\right\}=a, \min \left\{\mu_{i}, v_{i}\right\}=b$, we get

$$
\begin{aligned}
E(M(A, B)) & =\frac{1}{n} \sum_{i=1}^{n} \frac{1-\frac{1}{2}(a+b)+\frac{1}{2}(a-b)}{1+\frac{1}{2}(a-b)} \\
& =\frac{1}{n} \sum_{i=1}^{n} \frac{2-2 b}{2+(a-b)} \\
& =\frac{1}{n} \sum_{i=1}^{n} \frac{2-2 \min \left\{\mu_{i}, v_{i}\right\}}{2+\left|\mu_{i}-v_{i}\right|} .
\end{aligned}
$$


From Theorem 5, we obtain the similarity measure between $A$ and $B$ as following:

$$
\begin{aligned}
& S(A, B)=E(M(A, B)) \\
= & \frac{1}{n} \sum_{i=1}^{n} \frac{2-2 \min \left\{\left|\mu_{A}\left(x_{i}\right)-\mu_{B}\left(x_{i}\right)\right|,\left|v_{A}\left(x_{i}\right)-v_{B}\left(x_{i}\right)\right|\right\}}{2+|| \mu_{A}\left(x_{i}\right)-\mu_{B}\left(x_{i}\right)|-| v_{A}\left(x_{i}\right)-v_{B}\left(x_{i}\right)||} .
\end{aligned}
$$

Considering that the elements in the universe of discourse $X$ may have different importance, we define the weighted form of Formula (11).

Let $w=\left(w_{1}, w_{2}, \cdots, w_{n}\right)^{T}$ be a weighting vector of the elements $x_{i}(i=1,2, \cdots, n)$. Then the weighted similarity measure is defined as $S(A, B)=$

$$
\sum_{i=1}^{n} w_{i} \cdot \frac{2-2 \min \left\{\left|\mu_{A}\left(x_{i}\right)-\mu_{B}\left(x_{i}\right)\right|,\left|v_{A}\left(x_{i}\right)-v_{B}\left(x_{i}\right)\right|\right\}}{2+|| \mu_{A}\left(x_{i}\right)-\mu_{B}\left(x_{i}\right)|-| v_{A}\left(x_{i}\right)-v_{B}\left(x_{i}\right)||}
$$

where $w_{i} \in[0,1]$ and $\sum_{i=1}^{n} w_{i}=1 . \quad$ If $w=$ $\left(\frac{1}{n}, \frac{1}{n}, \cdots, \frac{1}{n}\right)^{T}$, then Formula (12) is reduced to Formula (11).

\subsection{Comparison of new similarity measure and some existing similarity measures}

In this subsection, the rationality of the new similarity measure will be demonstrated by the comparison between it and some existing similarity measures.

Let $A=\left\{\left\langle x_{i}, \mu_{A}\left(x_{i}\right), v_{A}\left(x_{i}\right)\right\rangle \mid x_{i} \in X\right\}$ and $B=$ $\left\{\left\langle x_{i}, \mu_{B}\left(x_{i}\right), v_{B}\left(x_{i}\right)\right\rangle \mid x_{i} \in X\right\}$ be two IFSs in the universe of discourse $X$. Chen ${ }^{43}$, Li and Cheng ${ }^{27}$ proposed the following similarity measures $S_{C}$ and $S_{D C}$ between the IFSs $A$ and $B$, respectively:

$$
\begin{gathered}
S_{C}(A, B)=1-\frac{\sum_{i=1}^{n}\left|\mu_{A}\left(x_{i}\right)-v_{A}\left(x_{i}\right)-\left(\mu_{B}\left(x_{i}\right)-v_{B}\left(x_{i}\right)\right)\right|}{2 n}, \\
S_{D C}(A, B)=1-\sqrt[p]{\frac{\sum_{i=1}^{n}\left|\psi_{A}\left(x_{i}\right)-\psi_{B}\left(x_{i}\right)\right|^{p}}{n}}
\end{gathered}
$$

where $p$ is a parameter with $1 \leqslant p<\infty$, and for each $i$,

$$
\begin{aligned}
& \psi_{A}\left(x_{i}\right)=\frac{\mu_{A}\left(x_{i}\right)+1-v_{A}\left(x_{i}\right)}{2}, \\
& \psi_{B}\left(x_{i}\right)=\frac{\mu_{B}\left(x_{i}\right)+1-v_{B}\left(x_{i}\right)}{2} .
\end{aligned}
$$

$\mathrm{Wei}^{36}, \mathrm{Xu}$ and Yager $^{30}$ presented similarity measures $S_{W W}$ and $S_{X Y}$, respectively.

$$
S_{W W}(A, B)=\frac{1}{n} \sum_{i=1}^{n} \frac{1-\min \left\{\mu_{i}, v_{i}\right\}}{1+\max \left\{\mu_{i}, v_{i}\right\}},
$$

where $\left|\mu_{A}\left(x_{i}\right)-\mu_{B}\left(x_{i}\right)\right|=\mu_{i},\left|v_{A}\left(x_{i}\right)-v_{B}\left(x_{i}\right)\right|=v_{i}$,

$$
S_{X Y}(A, B)=\frac{1}{n} \sum_{i=1}^{n} \frac{d\left(\alpha_{A}\left(x_{i}\right), \alpha_{B}^{c}\left(x_{i}\right)\right)}{d\left(\alpha_{A}, \alpha_{B}^{c}\right)+d\left(\alpha_{A}, \alpha_{B}\right)},
$$

where $\alpha_{A}\left(x_{i}\right)$ and $\alpha_{B}\left(x_{i}\right)$ are i-th IFNs of $A$ and $B$, respectively, and

$$
\begin{aligned}
d\left(\alpha_{A}\left(x_{i}\right), \alpha_{B}\left(x_{i}\right)\right) & =\frac{1}{2}\left(\left|\mu_{A}\left(x_{i}\right)-\mu_{B}\left(x_{i}\right)\right|\right. \\
& +\left|v_{A}\left(x_{i}\right)-v_{B}\left(x_{i}\right)\right| \\
& \left.+\left|\pi_{A}\left(x_{i}\right)-\pi_{B}\left(x_{i}\right)\right|\right) .
\end{aligned}
$$

Example 3. Let $A=\{\langle x, 0,0\rangle\}, \quad B=\{<$ $x, 0.5,0.5\rangle\}$ and $C=\{\langle x, 0.49,0.51\rangle\}$ be three IFSs. Using the similarity measures $S_{C}, S_{D C}$ and $S_{X Y}$, we get

$$
\begin{gathered}
S_{C}(A, B)=S_{D C}(A, B)=1.0000, \\
S_{C}(B, C)=S_{D C}(B, C)=0.9900, \\
S_{X Y}(A, B)=S_{X Y}(B, C)=0.5000 .
\end{gathered}
$$

Intuitively, we can see that the IFS $B$ is much more similar to $C$ than to $A$. However, $S_{C}(A, B)>S_{C}(B, C), S_{D C}(A, B)>S_{D C}(B, C)$, $S_{X Y}(A, B)=S_{X Y}(B, C)$, which are not consistent with our intuition.

Using the similarity measure $S_{W W}$ and the new similarity measure $S$, we derive

$$
\begin{gathered}
S_{W W}(A, B)=0.3333, S_{W W}(B, C)=0.9802, \\
S(A, B)=0.5000, S(B, C)=0.9900 .
\end{gathered}
$$

The results are so reasonable as we expect. Therefore, the similarity measure $S_{W W}$ and $S$ are more reasonable than $S_{C}, S_{D C}$ and $S_{X Y}$. 
$\mathrm{Li}$ and $\mathrm{Xu}^{44}$ established a new similarity measure $S_{L}$ as follows:

$$
\begin{aligned}
S_{L}(A, B) & =1-\frac{\sum_{i=1}^{n}\left|s_{A}\left(x_{i}\right)-s_{B}\left(x_{i}\right)\right|}{4 n} \\
& -\frac{\sum_{i=1}^{n}\left(\left|\mu_{A}\left(x_{i}\right)-\mu_{B}\left(x_{i}\right)\right|+\left|v_{A}\left(x_{i}\right)-v_{B}\left(x_{i}\right)\right|\right)}{4 n},
\end{aligned}
$$

where $s_{A}\left(x_{i}\right)=\mu_{A}\left(x_{i}\right)-v_{A}\left(x_{i}\right), \quad s_{B}\left(x_{i}\right)=\mu_{B}\left(x_{i}\right)-$ $v_{B}\left(x_{i}\right)$.

Xia and $\mathrm{Xu}^{29}$ defined a similarity measure $S_{X X}^{\eta, \kappa}$ :

$$
\begin{aligned}
S_{X X}^{\eta, \kappa}(A, B) & =1-\left(\frac{1}{n} \sum_{i=1}^{n} \mid(1-\kappa)\left(\mu_{A}\left(x_{i}\right)-\mu_{B}\left(x_{i}\right)\right)\right. \\
& \left.-\left.\kappa\left(v_{A}\left(x_{i}\right)-v_{B}\left(x_{i}\right)\right)\right|^{\eta}\right)^{\frac{1}{\eta}} .
\end{aligned}
$$

We further compare our similarity measure $S$ with $S_{C}, S_{D C}, S_{L}$ and $S_{X X}^{\eta, \kappa}$ by the following example.

Example 4. Let $A=\{\langle x, 0.4,0.2\rangle \mid x \in X\}, B=$ $\{<x, 0.5,0.3\rangle \mid x \in X\}$ and $C=\{\langle x, 0.5,0.2\rangle \mid x \in$ $X\}$ be three IFSs.

One can see intuitively that the IFS $A$ is more similar to $C$ than to $B$. Using the similarity measure $S_{C}, S_{D C}$ and $S_{L}$, however, we get that

$$
\begin{gathered}
S_{C}(A, B)=S_{D C}(A, B)=1.0000, \\
S_{C}(A, C)=S_{D C}(A, C)=0.9500, \\
S_{L}(A, B)=S_{L}(A, C)=0.9500,
\end{gathered}
$$

Thus, $S_{C}(A, B)>S_{C}(A, C), S_{D C}(A, B)>S_{D C}(A, C)$ and $S_{L}(A, B)=S_{L}(A, C)$, which are not consistent with our intuition.

Now, using $S_{X X}^{2,0.5}$ and our similarity measure $S$ given by Formula (11), we have

$$
\begin{gathered}
S_{X X}^{2,0.5}(A, B)=0.9000, S_{X X}^{2,0.5}(A, C)=0.9500, \\
S(A, B)=0.9000, S(A, C)=0.9524 .
\end{gathered}
$$

The results by the two measures $S_{X X}^{\eta, \kappa}$ and $S$ are similar and more consistent with our intuition.

\section{The applications of entropy and similarity measure}

In order to show the rationality and effectiveness of the new entropy and similarity measure proposed in Section 3 and 4, in this section, we apply them to multi-attribute group decision making with intuitionistic fuzzy information. The multi-attribute group decision making problem which is considered in this paper can be represented as follows.

Let $X=\left\{x_{1}, x_{2}, \cdots, x_{n}\right\}$ be a set of evaluation alternatives, $D=\left\{d_{1}, d_{2}, \cdots, d_{s}\right\}$ be a set of experts, $U=\left\{u_{1}, u_{2}, \cdots, u_{m}\right\}$ be an attribute set, $w=$ $\left(w_{1}, w_{2}, \cdots, w_{m}\right)^{T}$ be the weighting vector of attributes such that $w_{j} \in[0,1]$ and $\sum_{j=1}^{m} w_{j}=1$. Let $A_{k}=$ $\left(a_{i j}^{(k)}\right)_{n \times m}(k=1,2, \cdots, s)$ be intuitionistic fuzzy decision matrices where $a_{i j}^{(k)}=\left(t_{i j}^{(k)}, f_{i j}^{(k)}\right)$ is an IFV, provided by the decision maker $d_{k} \in D$ for the alternative $x_{i} \in X$ with respect to the attribute $u_{j} \in U$. Decision maker's goal is to obtain the ranking order of the alternatives.

According to Ref. 45, if attributes include cost attributes and benefit attributes in multi-attribute decision making process, we should transform the attribute values of cost type into those of benefit type. Hence decision making matrices $A_{k}=\left(a_{i j}^{(k)}\right)_{n \times m}(k=1,2, \cdots, s)$ are transformed into normalized decision making matrices $R_{k}=$ $\left(r_{i j}^{(k)}\right)_{n \times m}(k=1,2, \cdots, s)$ :

$r_{i j}^{(k)}=\left(\mu_{i j}^{(k)}, v_{i j}^{(k)}\right)=\left\{\begin{array}{l}a_{i j}^{(k)}, \text { for benefit attribute } u_{j}, \\ \bar{a}_{i j}^{(k)}, \text { for cost attribute } u_{j},\end{array}\right.$

where $\bar{a}_{i j}^{(k)}=\left(f_{i j}^{(k)}, t_{i j}^{(k)}\right), \pi_{i j}^{(k)}=1-t_{i j}^{(k)}-f_{i j}^{(k)}=$ $1-\mu_{i j}^{(k)}-v_{i j}^{(k)}(k=1,2, \cdots, s, i=1,2, \cdots, n, j=$ $1,2, \cdots, m)$.

For a given weighting vector of attributes, we can use the IFWA operator to derive the individual overall evaluation values $z_{i}^{(k)}=\left(\mu_{i}^{(k)}, v_{i}^{(k)}\right)$ of alterna- 
tives $x_{i}(i=1,2, \cdots, n)$ by experts $d_{k}(k=1,2, \cdots, s)$ :

$$
\begin{aligned}
z_{i}^{(k)} & =I F W A_{w}\left(r_{i 1}^{(k)}, r_{i 2}^{(k)}, \cdots, r_{i m}^{(k)}\right) \\
& =w_{1} r_{i 1}^{(k)} \oplus w_{2} r_{i 2}^{(k)} \oplus \cdots \oplus w_{m} r_{i m}^{(k)},
\end{aligned}
$$

where $w=\left(w_{1}, w_{2}, \cdots, w_{m}\right)^{T}$ is the weighting vector of the attributes of $u_{j}(j=1,2, \cdots, m)$, with $w_{j} \in$ $[0,1]$ and $\sum_{j=1}^{m} w_{j}=1$.

\subsection{Determining the weights of experts}

In order to fuse the evaluation values of all experts, we should determine the weights of experts. Sometimes, the information about weights of experts is completely unknown. Thus, it is a critical work to determine the objective weights of experts according to assessment information. $\mathrm{Xu}$ and $\mathrm{Cai}^{46}$ developed two nonlinear optimization models, one minimizing the divergence between each individual opinion and the group one, and the other minimizing the divergence among the individual opinions, to derive the weights of experts. In the following, we present two new methods, based on entropy and similarity measure, to determine the weights of experts.

During the decision making process, we usually expect that the uncertainty degrees of the decision results are as small as possible. Entropy can describe the uncertainty degree of intuitionistic fuzzy information. Let $z_{i}^{(k)}$ be individual overall evaluation value of alternative $x_{i} \in X$ by experts $d_{k} \in D$. The entropy for single element IFS $\left\{z_{i}^{(k)}\right\}=$ $\left\{\left(\mu_{i}^{(k)}\left(x_{i}\right), v_{i}^{(k)}\left(x_{i}\right)\right)\right\}$ is denoted by $E_{i k}$. Considering that the smaller entropy $E_{i k}$ is, the smaller uncertainty degree of $\left\{z_{i}^{(k)}\right\}$ is. Therefore, a reasonable weighting vector $\lambda=\left(\lambda_{1}, \lambda_{2}, \cdots, \lambda_{s}\right)^{T}$ should be determined so as to make all the uncertainty degrees of overall evaluation values for alternatives as small as possible. Based on this principle, we can establish the following multiple objective programming model:

$$
\begin{array}{ll}
\min & \left(\sum_{k=1}^{s} E_{1 k}^{2} \cdot \lambda_{k}^{2}, \sum_{k=1}^{s} E_{2 k}^{2} \cdot \lambda_{k}^{2}, \cdots, \sum_{k=1}^{s} E_{n k}^{2} \cdot \lambda_{k}^{2}\right) \\
\text { s.t. } & \left\{\begin{array}{l}
\sum_{k=1}^{s} \lambda_{k}=1 \\
\lambda_{k} \geqslant 0, \quad k=1,2, \cdots s .
\end{array}\right.
\end{array}
$$

where $\lambda=\left(\lambda_{1}, \lambda_{2}, \cdots, \lambda_{s}\right)^{T}$ is the weighting vector of $d_{k}(k=1,2, \cdots, s)$.

We can transform the multiple objective programming model into a single objective optimization model:

$$
\begin{array}{ll}
\min & \sum_{i=1}^{n} \sum_{k=1}^{s} E_{i k}^{2} \cdot \lambda_{k}^{2} \\
\text { s.t. } & \left\{\begin{array}{l}
\sum_{k=1}^{s} \lambda_{k}=1 \\
\lambda_{k} \geqslant 0, \quad k=1,2, \cdots s,
\end{array}\right.
\end{array}
$$

where $\lambda=\left(\lambda_{1}, \lambda_{2}, \cdots, \lambda_{s}\right)^{T}$ is the weighting vector of $d_{k}(k=1,2, \cdots, s)$.

To solve this model, we construct the Lagrange function

$$
L(\lambda, \xi)=\sum_{i=1}^{n} \sum_{k=1}^{s} E_{i k}^{2} \cdot \lambda_{k}^{2}+2 \xi\left(\sum_{k=1}^{s} \lambda_{k}-1\right),
$$

where $\xi$ is the Lagrange multiplier.

Differentiating $L(\lambda, \xi)$ with respect to $\lambda_{k}(k=$ $1,2, \cdots, s)$ and $\xi$, and setting these partial derivatives equal to zero, we obtain the following equations:

$$
\left\{\begin{array}{l}
\frac{\partial L}{\partial \lambda_{k}}=2 \lambda_{k} \cdot \sum_{i=1}^{n} E_{i k}^{2}+2 \xi=0, \quad k=1,2, \cdots, s . \\
\frac{\partial L}{\partial \xi}=2 \sum_{k=1}^{s} \lambda_{k}-2=0 .
\end{array}\right.
$$

By solving equations above, we get the weights of experts as follows:

$$
\lambda_{k}^{(1)}=\frac{1}{\sum_{i=1}^{n} E_{i k}^{2} \cdot \sum_{k=1}^{s} \frac{1}{\sum_{i=1}^{n} E_{i k}^{2}}}, \quad k=1,2, \cdots s .
$$

In the following, we determine the weights of experts from another point of view. Let $z_{i}^{(k)}=$ $\left(\mu_{i}^{(k)}, v_{i}^{(k)}\right)(i=1,2, \cdots, n)$ be the individual overall evaluation values of $x_{i}(i=1,2, \cdots, n)$ by the expert $d_{k} \in D$. Hence $Z_{k}=\left\{z_{i}^{(k)} \mid i=1,2, \cdots, n\right\}$ is an intuitionistic fuzz set. We define ideal alternatives set 
$Z^{+}=\left\{z_{i}^{+} \mid i=1,2, \cdots, n\right\}$ and anti-ideal alternatives set $Z^{-}=\left\{z_{i}^{-} \mid i=1,2, \cdots, n\right\}$, where

$$
\begin{gathered}
z_{i}^{+}=\bigcup_{1 \leqslant k \leqslant s} z_{i}^{(k)}=\left(\max _{1 \leqslant k \leqslant s}\left\{\mu_{i}^{(k)}\right\}, \min _{1 \leqslant k \leqslant s}\left\{v_{i}^{(k)}\right\}\right), \\
z_{i}^{-}=\bigcap_{1 \leqslant k \leqslant s} z_{i}^{(k)}=\left(\min _{1 \leqslant k \leqslant s}\left\{\mu_{i}^{(k)}\right\}, \max _{1 \leqslant k \leqslant s}\left\{v_{i}^{(k)}\right\}\right) .
\end{gathered}
$$

The similarity degrees between $Z_{k}$ and $Z^{+}, Z_{k}$ and $Z^{-}$are defined as:

$$
\begin{aligned}
& S_{k}^{+}=S\left(Z_{k}, Z^{+}\right), \quad k=1,2, \cdots, s, \\
& S_{k}^{-}=S\left(Z_{k}, Z^{-}\right), \quad k=1,2, \cdots, s .
\end{aligned}
$$

Then we define the averaging alternatives set $Z^{*}=\left\{z_{i}^{*} \mid i=1,2, \cdots, n\right\}$, where

$$
z_{i}^{*}=\frac{1}{s}\left(z_{i}^{(1)} \oplus z_{i}^{(2)} \oplus \cdots \oplus z_{i}^{(s)}\right), \quad i=1,2, \cdots, n .
$$

The similarity degrees between $Z_{k}$ and $Z^{*}$ is defined as:

$$
S_{k}^{*}=S\left(Z_{k}, Z^{*}\right), \quad k=1,2, \cdots, s .
$$

The ideal alternatives set and anti-ideal alternatives set reflect the extreme views of experts, while the averaging alternatives set reflects the group views of experts. In group decision making process, we usually expect to reach with a high group consensus. Thus, the smaller $S_{k}^{+}$and $S_{k}^{-}$are, the bigger weight is given to the expert $d_{k}$; the bigger $S_{k}^{*}$ is, the bigger weight is given to the expert $d_{k}$. Therefore, we define the weights of experts $d_{k}(k=1,2, \cdots, s)$ as follows:

$\lambda_{k}^{(2)}=\frac{\lambda_{k}^{*}}{\sum_{k=1}^{s} \lambda_{k}^{*}}$, where $\lambda_{k}^{*}=\frac{S_{k}^{*}}{S_{k}^{+}+S_{k}^{-}}, k=1,2, \cdots, s$.

Based on different perspectives, we derive the weighting vectors $\lambda^{(1)}$ and $\lambda^{(2)}$. Then we can integrate them into a combined weighting vector $\lambda=\left(\lambda_{1}, \lambda_{2}, \cdots, \lambda_{s}\right)^{T}$, where $\lambda_{k}=\alpha \lambda_{k}^{(1)}+(1-$ $\alpha) \lambda_{k}^{(2)}(k=1,2, \cdots, s)$, and $\alpha \in[0,1]$ reflects decision maker's subjective preference.

\subsection{A multi-attribute group decision making approach with intuitionistic fuzzy information}

Based on the IFWA operator and two methods to determine weights of experts in Section 5.1, we can describe the following steps to get the ranking of alternatives.

Step 1 Utilize Formula (14) to transform decision making matrices $A_{k}=\left(a_{i j}^{(k)}\right)_{n \times m}$ into normalized decision making matrices $R_{k}=\left(r_{i j}^{(k)}\right)_{n \times m}$.

Step 2 Utilize Formula (15) to derive the individual overall evaluation values $z_{i}^{(k)}(i=$ $1,2, \cdots, n, k=1,2, \cdots, s)$ of alternatives $x_{i}(i=$ $1,2, \cdots, n)$ by experts $d_{k}(k=1,2, \cdots, s)$.

Step 3 Utilize Formula (20) to derive experts' weighting vector $\lambda^{(1)}=\left(\lambda_{1}^{(1)}, \lambda_{2}^{(1)}, \cdots, \lambda_{s}^{(1)}\right)^{T}$; utilize Formulas (21)-(26) to derive experts' weighting vector $\lambda^{(2)}=\left(\lambda_{1}^{(2)}, \lambda_{2}^{(2)}, \cdots, \lambda_{s}^{(2)}\right)^{T}$.

Step 4 Integrate the weighting vectors $\lambda^{(1)}$ and $\lambda^{(2)}$ into the objective experts' weighting vector $\lambda=$ $\left(\lambda_{1}, \lambda_{2}, \cdots, \lambda_{s}\right)^{T}$, where $\lambda_{k}=\alpha \lambda_{k}^{(1)}+(1-\alpha) \lambda_{k}^{(2)}$, $\alpha \in[0,1], k=1,2, \cdots, s$.

Step 5 Utilize the IFWA operator to derive the overall evaluation values $z_{i}(i=1,2, \cdots, n)$ of the alternatives $x_{i}(i=1,2, \cdots, n)$ :

$$
\begin{aligned}
z_{i} & =\operatorname{IFW} A_{\lambda}\left(z_{i}^{(1)}, z_{i}^{(2)}, \cdots z_{i}^{(s)}\right) \\
& =\lambda_{1} z_{i}^{(1)} \oplus \lambda_{2} z_{i}^{(2)} \oplus \cdots \oplus \lambda_{s} z_{i}^{(s)},
\end{aligned}
$$

where $\lambda=\left(\lambda_{1}, \lambda_{2}, \cdots, \lambda_{s}\right)^{T}$ is the weighting vector of experts with $\lambda_{k} \in[0,1]$ and $\sum_{k=1}^{s} \lambda_{k}=1$.

Step 6 Utilize Definition 4 to compare the overall evaluation values $z_{i}(i=1,2, \cdots, n)$ and rank the alternatives $x_{i}(i=1,2, \cdots, n)$.

In order to verify effectiveness of the proposed decision making approach, two instances, adapted from $\mathrm{Xu}^{46}$ and $\mathrm{Wan}^{47}$, are provided as follows.

Example 5. ${ }^{46}$ Consider an air-condition system selection problem. Suppose that there exist three air-condition systems $x_{i}(i=1,2,3)$ to be selected, and the following is the list of five attribute $u_{j}(j=$ $1,2,3,4,5)$ : good quality $\left(u_{1}\right)$, easy to operate $\left(u_{2}\right)$, economical $\left(u_{3}\right)$, good service after selling $\left(u_{4}\right)$ 
and cost $\left(u_{5}\right)$. Among these attributes, $u_{j}(j=$ $1,2,3,4)$ are of benefit type, $u_{5}$ is of cost type. $w=$ $(0.200,0.299,0.106,0.156,0.239)^{T}$ is the weighting vector of attributes. An experts group, which consists 3 experts $d_{k}(k=1,2,3)$. These experts $d_{k}(k=$ $1,2,3)$ evaluate the air-condition systems $x_{i}(i=$ $1,2,3)$ by the IFNs $a_{i j}^{(k)}=\left(t_{i j}^{(k)}, f_{i j}^{(k)}\right)(i=1,2,3, j=$ $1,2,3,4,5, k=1,2,3)$ with respect to the attributes $u_{j}(j=1,2,3,4,5)$. The decision making matrices $A_{k}=\left(a_{i j}^{(k)}\right)_{3 \times 5}(k=1,2,3)$ are as follows:

$$
\begin{gathered}
\left(\begin{array}{lllll}
(0.8,0.1) & (0.7,0.1) & (0.7,0.2) & (0.9,0.0) & (0.4,0.5) \\
(0.7,0.1) & (0.8,0.2) & (0.6,0.4) & (0.7,0.1) & (0.6,0.4) \\
(0.8,0.2) & (0.9,0.1) & (0.7,0.0) & (0.7,0.2) & (0.5,0.5)
\end{array}\right) \\
\left(\begin{array}{lllll}
(0.9,0.1) & (0.8,0.1) & (0.7,0.0) & (0.9,0.1) & (0.3,0.7) \\
(0.7,0.2) & (0.8,0.1) & (0.9,0.1) & (0.7,0.3) & (0.7,0.2) \\
(0.7,0.1) & (0.9,0.0) & (0.8,0.0) & (0.8,0.2) & (0.6,0.3)
\end{array}\right) \\
\left(\begin{array}{lllll}
(0.8,0.0) & (0.7,0.1) & (0.9,0.0) & (0.8,0.1) & (0.4,0.6) \\
(0.8,0.2) & (0.7,0.3) & (0.8,0.1) & (0.9,0.1) & (0.6,0.3) \\
(0.9,0.1) & (0.8,0.0) & (0.8,0.1) & (0.9,0.0) & (0.5,0.4)
\end{array}\right)
\end{gathered}
$$

Step 1 Utilize Formula (14) to transform decision making matrices $A_{k}=\left(a_{i j}^{(k)}\right)_{3 \times 5}(k=1,2,3)$ into normalized decision making matrices $R_{k}=$ $\left(r_{i j}^{(k)}\right)_{3 \times 5}(k=1,2,3)$.

$\left(\begin{array}{lllll}(0.8,0.1) & (0.7,0.1) & (0.7,0.2) & (0.9,0.0) & (0.5,0.4) \\ (0.7,0.1) & (0.8,0.2) & (0.6,0.4) & (0.7,0.1) & (0.4,0.6) \\ (0.8,0.2) & (0.9,0.1) & (0.7,0.0) & (0.7,0.2) & (0.5,0.5)\end{array}\right)$
$\left(\begin{array}{ccccc}(0.9,0.1) & (0.8,0.1) & (0.7,0.0) & (0.9,0.1) & (0.7,0.3) \\ (0.7,0.2) & (0.8,0.1) & (0.9,0.1) & (0.7,0.3) & (0.2,0.7) \\ (0.7,0.1) & (0.9,0.0) & (0.8,0.0) & (0.8,0.2) & (0.3,0.6)\end{array}\right)$
$\left(\begin{array}{ccccc}(0.8,0.0) & (0.7,0.1) & (0.9,0.0) & (0.8,0.1) & (0.6,0.4) \\ (0.8,0.2) & (0.7,0.3) & (0.8,0.1) & (0.9,0.1) & (0.3,0.6) \\ (0.9,0.1) & (0.8,0.0) & (0.8,0.1) & (0.9,0.0) & (0.4,0.5)\end{array}\right)$

Step 2 Utilize Formula (15) to derive the individual overall evaluation values $z_{i}^{(k)}(i=1,2,3, k=$ $1,2,3)$ of alternatives $x_{i}(i=1,2,3)$ by experts $d_{k}(k=1,2,3)$.

$$
\begin{aligned}
& z_{1}^{(1)}=(0.7367,0.0000), z_{2}^{(1)}=(0.6767,0.2187), \\
& z_{3}^{(1)}=(0.7750,0.0000), z_{1}^{(2)}=(0.8203,0.0000),
\end{aligned}
$$

$$
\begin{aligned}
& z_{2}^{(2)}=(0.7010,0.2171), z_{3}^{(2)}=(0.7622,0.0000), \\
& z_{1}^{(3)}=(0.7524,0.0000), z_{2}^{(3)}=(0.7266,0.2448), \\
& z_{3}^{(3)}=(0.7968,0.0000) .
\end{aligned}
$$

Step 3 Utilize Formulas (9) and (20) to derive experts' weighting vector $\lambda^{(1)}=$ $(0.297,0.354,0.349)^{T}$, utilize Formulas (11), (21)(26) to derive experts' weighting vector $\lambda^{(2)}=$ $(0.334,0.333,0.333)^{T}$.

Step 4 Integrate the weighting vectors $\lambda^{(1)}$ and $\lambda^{(2)}$ into the objective experts' weighting vector $\lambda=(0.315,0.344,0.341)^{T}$, where $\lambda_{k}=\alpha \lambda_{k}^{(1)}+$ $(1-\alpha) \lambda_{k}^{(2)}, \alpha=0.5, k=1,2,3$.

Step 5. Utilize the Formula (27) to derive the overall evaluation values $z_{i}(i=1,2,3)$ of the alternatives $x_{i}(i=1,2,3)$ :

$$
\begin{aligned}
& z_{1}=I F W A_{\lambda}\left(z_{1}^{(1)}, z_{1}^{(2)}, z_{1}^{(3)}\right)=(0.7739,0.0000) ; \\
& z_{2}=I F W A_{\lambda}\left(z_{2}^{(1)}, z_{2}^{(2)}, z_{2}^{(3)}\right)=(0.7028,0.2267) ; \\
& z_{3}=I F W A_{\lambda}\left(z_{3}^{(1)}, z_{3}^{(2)}, z_{3}^{(3)}\right)=(0.7785,0.0000) .
\end{aligned}
$$

Step 6 Utilize the score function to calculate the scores $s\left(z_{i}\right)(i=1,2,3)$ of overall evaluation values $z_{i}(i=1,2,3)$ of the alternatives $x_{i}(i=1,2,3)$ :

$$
s\left(z_{1}\right)=0.7739, s\left(z_{2}\right)=0.4761, s\left(z_{3}\right)=0.7785 .
$$

Utilize the score degrees $s\left(z_{i}\right)(i=1,2,3)$ to rank the alternatives $x_{i}(i=1,2,3)$, we obtain

$$
x_{3} \succ x_{1} \succ x_{2} .
$$

Therefore, $x_{3}$ is the best alternative.

Example 6. ${ }^{47}$ A manufacturing company search the best global supplier for one of its most critical parts used in assembling process. The attributes which are considered here in selection of three suppliers $x_{i}(i=1,2,3)$ are: capacity of the production $\left(u_{1}\right)$, capacity of accuracy $\left(u_{2}\right)$, supplier's credibility $\left(u_{3}\right)$, cost of the product $\left(u_{4}\right)$. Among these attributes, $u_{j}(j=1,2,3)$ are of benefit type, $u_{4}$ is of cost type. $\quad w=(0.31,0.42,0.16,0.11)^{T}$ is the weighting vector of the attributes. An experts group is formed which consists of three experts $d_{k}(k=1,2,3)$ (whose weighting vector is to be determined). The experts $d_{k}(k=1,2,3)$ represent the characteristics of the suppliers $x_{i}(i=1,2,3)$ by the 
IFNs $a_{i j}^{(k)}(i=1,2,3, j=1,2,3,4, k=1,2,3)$ with respect to the attributes $u_{j}(j=1,2,3,4)$. The decision making matrices $A_{k}=\left(r_{i j}^{(k)}\right)_{3 \times 4}(k=1,2,3)$ are as follows:

$$
\begin{aligned}
& A_{1}=\left(\begin{array}{llll}
(0.4,0.2) & (0.1,0.4) & (0.3,0.6) & (0.1,0.6) \\
(0.2,0.5) & (0.3,0.6) & (0.3,0.5) & (0.3,0.5) \\
(0.5,0.3) & (0.4,0.5) & (0.3,0.6) & (0.7,0.2)
\end{array}\right) \\
& A_{2}=\left(\begin{array}{llll}
(0.5,0.3) & (0.3,0.6) & (0.3,0.4) & (0.4,0.5) \\
(0.4,0.3) & (0.3,0.5) & (0.2,0.6) & (0.3,0.5) \\
(0.3,0.5) & (0.4,0.5) & (0.5,0.3) & (0.3,0.6)
\end{array}\right) \\
& A_{3}=\left(\begin{array}{llll}
(0.2,0.4) & (0.5,0.3) & (0.4,0.6) & (0.3,0.4) \\
(0.4,0.5) & (0.3,0.6) & (0.2,0.5) & (0.7,0.1) \\
(0.3,0.6) & (0.4,0.4) & (0.3,0.5) & (0.4,0.5)
\end{array}\right)
\end{aligned}
$$

Step 1. Utilize Formula (14) to transform decision making matrices $A_{k}=\left(a_{i j}^{(k)}\right)_{3 \times 4}(k=1,2,3)$ into normalized decision making matrices $R_{k}=$ $\left(r_{i j}^{(k)}\right)_{3 \times 4}(k=1,2,3)$.

$R_{1}=\left(\begin{array}{llll}(0.4,0.2) & (0.1,0.4) & (0.3,0.6) & (0.6,0.1) \\ (0.2,0.5) & (0.3,0.6) & (0.3,0.5) & (0.5,0.3) \\ (0.5,0.3) & (0.4,0.5) & (0.3,0.6) & (0.2,0.7)\end{array}\right)$

$R_{2}=\left(\begin{array}{cccc}(0.5,0.3) & (0.3,0.6) & (0.3,0.4) & (0.5,0.4) \\ (0.4,0.3) & (0.3,0.5) & (0.2,0.6) & (0.5,0.3) \\ (0.3,0.5) & (0.4,0.5) & (0.5,0.3) & (0.6,0.3)\end{array}\right)$

$R_{3}=\left(\begin{array}{cccc}(0.2,0.4) & (0.5,0.3) & (0.4,0.6) & (0.4,0.3) \\ (0.4,0.5) & (0.3,0.6) & (0.2,0.5) & (0.1,0.7) \\ (0.3,0.6) & (0.4,0.4) & (0.3,0.5) & (0.5,0.4)\end{array}\right)$

Step 2. Utilize Formula (15) to derive the individual overall evaluation values $z_{i}^{(k)}(i=1,2,3, k=$ $1,2,3)$ of alternatives $x_{i}(i=1,2,3)$ by experts $d_{k}(k=1,2,3)$.

$$
\begin{aligned}
& z_{1}^{(1)}=(0.3026,0.2956), z_{2}^{(1)}=(0.2969,0.5103), \\
& z_{3}^{(1)}=(0.4001,0.4560), z_{1}^{(2)}=(0.3923,0.4338), \\
& z_{2}^{(2)}=(0.3430,0.4154), z_{3}^{(2)}=(0.4154,0.4356), \\
& z_{1}^{(3)}=(0.3924,0.3665), z_{2}^{(3)}=(0.2991,0.5601), \\
& z_{3}^{(3)}=(0.3677,0.4701) .
\end{aligned}
$$

Step 3. Utilize Formulas (9) and (20) to derive experts' weighting vector $\lambda^{(1)}=$
$(0.333,0.308,0.359)^{T}$; utilize Formulas (11), (21)(26) to derive experts' weighting vector $\lambda^{(2)}=$ $(0.335,0.330,0.335)^{T}$.

Step 4. Integrate the weighting vector $\lambda^{(1)}$ and $\lambda^{(2)}$ into an objective experts' weighting vector $\lambda=(0.334,0.319,0.347)^{T}$, where $\lambda_{k}=\alpha \lambda_{k}^{(1)}+$ $(1-\alpha) \lambda_{k}^{(2)}, \alpha=0.5, k=1,2,3$.

Step 5. Utilize the Formula (27) to derive the overall evaluation values $z_{i}(i=1,2,3)$ of the alternatives $x_{i}(i=1,2,3)$ :

$$
\begin{aligned}
& z_{1}=I F W A_{\lambda}\left(z_{1}^{(1)}, z_{1}^{(2)}, z_{1}^{(3)}\right)=(0.3637,0.3600) ; \\
& z_{2}=I F W A_{\lambda}\left(z_{2}^{(1)}, z_{2}^{(2)}, z_{2}^{(3)}\right)=(0.3127,0.4936) ; \\
& z_{3}=I F W A_{\lambda}\left(z_{3}^{(1)}, z_{3}^{(2)}, z_{3}^{(3)}\right)=(0.3941,0.4542) .
\end{aligned}
$$

Step 6. Utilize the score function to calculate the scores $s\left(z_{i}\right)(i=1,2,3)$ of overall evaluation values $z_{i}(i=1,2,3)$ of the alternatives $x_{i}(i=1,2,3)$ :

$s\left(z_{1}\right)=0.0038, s\left(z_{2}\right)=-0.1809, s\left(z_{3}\right)=-0.0601$.

Utilize the score degrees $s\left(z_{i}\right)(i=1,2,3)$ to rank the alternatives $x_{i}(i=1,2,3)$, we obtain

$$
x_{1} \succ x_{3} \succ x_{2} .
$$

Therefore, we obtain that $x_{1}$ is the best alternative.

\section{Conclusions}

Entropy and similarity measure are two important notions for intuitionistic fuzzy sets. To improve the entropy measure provided by Szmidt and Kacprzyk $^{26}$, we have established a new entropy measure with geometrical interpretation of intuitionistic fuzzy sets. The new entropy formula can measure both fuzziness and intuitionism of intuitionistic fuzzy sets. According to the relationship between entropy and similarity measure, we have presented a new similarity measure between two intuitionistic fuzzy sets. Based on entropy and similarity measures, we have proposed two methods to determine weights of experts for multi-attribute group decision making under intuitionistic fuzzy environment. Finally, we have established a multi-attribute group decision making method and adopt illustrative examples to demonstrate its rationality. 


\section{Acknowledgments}

The authors are very grateful to the editor and anonymous referees for their insightful and valuable suggestions that have led to an improved version of this paper.

\section{References}

1. L. A. Zadeh, Fuzzy sets, Information and Control, 8, 338-356 (1965).

2. K. Atanassov, Intuitionistic fuzzy sets, Fuzzy Sets and System, 20(1), 87-96 (1986).

3. Z. S. Xu, Methods for aggregating interval-valued intuitionistic fuzzy information and their application to decision making, Control and Decision, 22(2), 215219 (2007).

4. Z. S. Xu, Multiple attribute decision making with intuitionistic fuzzy preference information, Systems Engineering-Theory \& Practice, 27(11), 62-71 (2007).

5. L. A. Zadeh, The concept of a linguistic variable and its application to approximate reasoning-I, Information Sciences, 8, 199-249 (1975).

6. W. L. Gau and D. J. Buehrer, Vague sets, IEEE Transactions on systems, Man and Cybernetics, 23(2), 610614 (1993).

7. P. Burillo and H. Bustince, Vague sets are intuitionistic sets, Fuzzy Sets and Systems, 79, 403-405 (1996).

8. C. Cornelis, K. Atanassov and E. E. Kerre, Intuitionistic fuzzy sets and interval-valued fuzzy sets: a critical comparison, In: Proc. Third European Conf. on Fuzzy Logic and Technol(EUSFLAT'03), Zittau, Germany, pp. 159-163 (2003).

9. G. Deschrijver and E. E. Kerre, On the relationship between some extensions of fuzzy set theory, Fuzzy Sets and Systems, 133, 227-235 (2003).

10. Z. S. Xu, A deviation-based approach to intuitionistic fuzzy multiple attribute group decision making, Group Decision and Negotiation, 19, 57-76 (2010).

11. Z. S. Xu, Approaches to multiple attribute group decision making based on intuitionistic fuzzy power aggregation operators, Knowledge-Based Systems, 24, 749-760 (2011).

12. Z. S. Xu and R. R. Yager, Dynamic intuitionistic fuzzy multi-attribute decision making, International Journal of Approximate Reasoning, 48, 246-262 (2008).

13. C. P. Wei and X. J. Tang, A fuzzy group decision making approach based on entropy, similarity measure, Int. J. of Information Technology \& Decision Making, 10, 1111-1130 (2011).

14. S. K. De, R. Biswas and A. R. Roy, An application of intuitionistic fuzzy sets in medical diagnosis, Fuzzy
Sets and Systems, 117(2), 209-213 (2001).

15. I. K. Vlachos and G. D. Sergiadis, Intuitionistic fuzzy information-applications to pattern recognition, Pattern Recognition Letters, 28, 197-206 (2007).

16. Z. S. Xu, On similarity measures of interval-valued intuitionistic fuzzy sets and their application to pattern recognitions, Journal of Southeast University(English Edition), 23(1), 139-143 (2007).

17. Z. S. Xu, J. Chen and J. J. Wu, Clustering algorithm for intuitionistic fuzzy sets, Information Sciences, 178, 3775-3790 (2008).

18. L. A. Zadeh, Fuzzy sets and systems, Proceedings of the Symposium on Systems Theory, Polytechnic Institute of Brooklyn, New York, 29-37 (1965).

19. L. A. De and S. Termini, A definition of nonprobabilistic entropy in the setting of fuzzy sets theory, Information and Control, 20, 301-312 (1972).

20. P. Burillo and H. Bustince, Entropy on intuitionistic fuzzy sets and on interval-valued fuzzy sets, Fuzzy Sets and Systems, 78, 305-316 (1996).

21. E. Szmidt and J. Kacprzyk, Entropy for intuitionistic fuzzy sets, Fuzzy Sets and Systems, 118(3), 467-477 (2001).

22. C. P. Wei, Z. H. Gao and T. T. Guo, An intuitionistic fuzzy entropy measure based on the trigonometric function, Control and Decision, 27(4), 571-574 (2012)(in Chinese).

23. M. M. Xia and Z. S. Xu, Entropy/cross entropy-based group decision making under intuitionistic fuzzy environment, Information Fusion, 13(1), 31-47 (2012).

24. I. K. Vlachos and G. D. Sergiadis, Subsethood, entropy, and cardinality for interval-valued fuzzy setsAn algebraic derivation, Fuzzy Sets and Systems, 158, 1384-1396 (2007).

25. Q. S. Zhang and S. Y. Jiang, A note on information entropy measures for vague sets and its application, Information Sciences, 178, 4184-4191 (2008).

26. E. Szmidt and J. Kacprzyk, New measures of entropy for intuitionistic fuzzy sets, Ninth Int. Conf. on IFSs, Sofia, 11(2), 12-20 (2005).

27. D. F. Li and C. T. Cheng, New similarity measure of intuitionistic fuzzy sets and application to pattern recongnitions, Pattern Recognition Letters, 23, 221-225 (2002).

28. Z. Z. Liang and P. F. Shi, Similarity measures on intuitionistic fuzzy sets, Pattern Recognition Letters, 24 2687-2693 (2003).

29. M. M. Xia and Z. S. Xu, Some new similarity measures for intuitionistic fuzzy values and their application in group decision making, Journal of Systems Science and Systems Engineering, 19(4), 430452 (2011).

30. Z. S. Xu and R. R. Yager, Intuitionistic and intervalvalued intutionistic fuzzy preference relations and their measures of similarity for the evaluation of 
agreement within a group, Fuzzy Optimization and Decision Making, 8, 123-139 (2009).

31. Y. H. Li, D. L. Olson and Q. Zeng, Similarity measures between intuitionistic fuzzy(Vague) set: a comparative analysis, Pattern Recognition Letters, 28, 278-285 (2007)

32. Z. S. Xu, An overview of distance and similarity measures of intuitionistic sets, International Journal of Uncertainty, Fuzziness and Knowledge-Based Systems, 16(4), 529-555 (2008)

33. W. Y. Zeng and H. X. Li, Relationship between similarity measure and entropy of interval-valued fuzzy sets, Fuzzy Sets and Systems, 157, 1477-1484 (2006).

34. H. Y. Zhang, W. X. Zhang and C. L. Mei, Entropy of interval-valued fuzzy sets based on distance and its relationship with similarity measure, Knowledge-Based Systems, 22, 449-454 (2009)

35. W. Y. Zeng and P. Guo, Normalized distance, similarity measure, inclusion measure and entropy of interval-valued fuzzy sets and their relationship, Information Sciences, 178, 1334-1342 (2008).

36. C. P. Wei, P. Wang and Y. Z. Zhang, Entropy, Similarity Measure of Interval-valued Intuitionistic Fuzzy Sets and Their Applications, Information Sciences, 182(19), 4273-4286 (2011).

37. E. Szmidt and J. Kacprzyk, Distance between intuitionistic fuzzy sets, Fuzzy Sets and Systems, 114, 505518 (2000).

38. Z. S. Xu and R. R. Yager, Some geometric aggregation operators based on intuitionistic fuzzy sets, International Journal of General System, 35(4), 417-433
(2006).

39. S. M. Chen and J. M. Tan, Handling multi-criteria fuzzy decision making problems based on vague set theory, Fuzzy Sets and Systems, 67(2), 163-172 (1994).

40. D. H. Hong and C. H. Choi, Multicriteria fuzzy decision-making problems based on vague set theory, Fuzzy Sets and Systems, 114, 103-113 (2000)

41. Z. S. Xu, Intuitionistic fuzzy aggregation operators, IEEE Transactions on Fuzzy Systems, 15, 1179-1187 (2007).

42. H. B. Mitchell, On the Dengfeng-Chuntain similarity measure and its application to pattern recognition, Pattern Recognition Letters, 24, 3101-3104 (2003).

43. S. M. Chen, Measures of similarity between vague sets, Fuzzy Sets and Systems, 74, 217-223 (1995).

44. F. Li and Z. Y. Xu, Similarity measures between vague sets, Software, 12(6), 922-927 (2001)(in Chinese).

45. Z. S. Xu and H. Hu, Projection models for intuitionistic fuzzy multiple attribute decision making, International Journal of Information Technology and Decision Making, 9(2), 267-280 (2010).

46. Z. S. Xu and X. Q. Cai, Nonlinear optimization models for multiple attribute group decision making with intuitionistic fuzzy information, International Journal of Intelligent Systems, 25, 489-513 (2010).

47. S. P. Wan, Determination of experts' weights based on Vague sets for multi-attribute group decision-making, Communication on Applied Mathematics and Computation, 24(1), 45-52 (2010). 